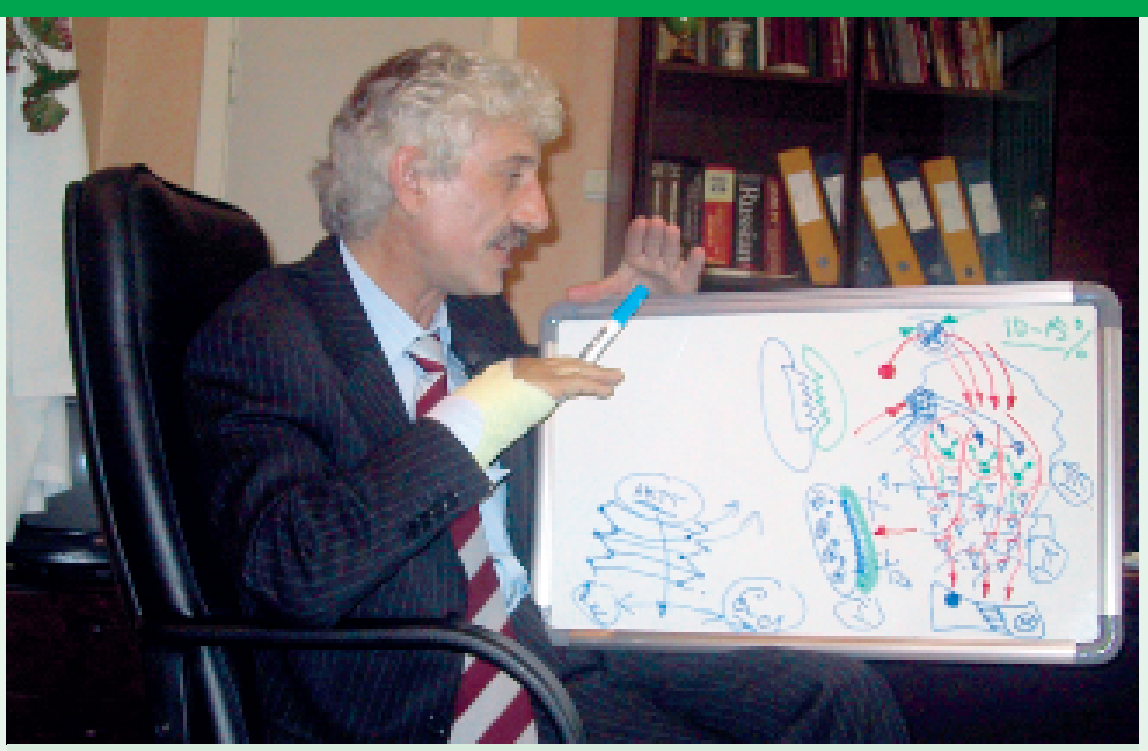

Der russische Arzt Michail Zobin erläutert sein Suchtmodell und die Wirkungsweise seiner Behandlung.

\title{
Die «Transmodulationsblockade» oder «Zobin-Methode»
}

Robert Hämmig, wie Rudolf Stohler und Andreas Moldovanyi ein Experte auf dem Gebiet der substanzgebundenen Abhängigkeitserkrankungen, hat im März gemeinsam mit Journalistenteams einen opioidabhängigen Patienten nach Moskau begleitet und dessen Behandlung durch den russischen Arzt Dr. Zobin beobachtet. Im folgenden schildert er seine Eindrücke unter Bezugnahme auf die Aussagen seiner Kollegen in der SÄZ Nr. 14/2006.

Korrespondenz

Dr. med. Robert Hämmig Universitäre Psychiatrische Dienste Bern

Direktion Psychiatrie Murtenstrasse 21 / Pf 52 CH-3010 Bern

haemmig@spk.unibe.ch
Ex cathedra haben meine beiden langjährigen Kollegen, Freunde und Mitstreiter in der Verbesserung des Schicksals von Drogenabhängigen natürlich recht. Nach der Durchsicht der von ihnen zitierten Webseiten bin ich zum gleichen Schluss gekommen: unzulängliche Heilsversprechungen, wie wir sie seit Jahrzehnten in bezug auf Drogentherapien kennen («Cold Turkey», «Blitzentzug», «Naltrexon-Therapie», «Brechkuren» bei thailändischen Mönchen usw., weitere sind im Anzug wie vietnamesische und chinesische Behandlungen).

Anders wohl als meine Kollegen hat mich aber die Neugier gepackt, und ich habe die Einladung angenommen, an der «therapeutischen Gruppenreise», bestehend aus einem Patienten, zwei Begleitpersonen seines Vertrauens, zwei freien Mitarbeitern des Fernsehens und mir, teilzunehmen. Da ich mir bewusst bin, dass unsere von englischer Literatur dominierte Schulmedizin nur ein Subsystem einer globalen Medizin darstellt, hoffte ich, auf dieser Reise einen kleinen Einblick in das exsowjetische Schulmedizinsystem zu erhalten. Zudem konnte ich mir aufgrund der zitierten Webseiten kein richtiges Bild von der Behandlung machen. Das Rätsel der unverständlichen Webtexte hat seine Lösung darin, dass sie von Nicole Gyr, der Frau, die selbst die Behandlung gemacht hat und aufgrund ihrer eigenen positiven Erfahrung das Verfahren jetzt propagiert, verfasst wurden. Sie ist Laie und wäre sehr froh, fachliche Unterstützung bei der Verfassung der Beschreibungen zu erhalten. 


\section{Belesener suchtpsychiatrischer Fachkollege}

Die Begegnung mit Zobin hat mich sehr überrascht. In längeren Fachgesprächen lernte ich Michail Zobin als belesenen suchtpsychiatrischen Fachkollegen kennen. Er ist dezidiert der Meinung, dass die russische Regierung so schnell wie möglich Methadon von der Liste der verbotenen Substanzen auf die der verschreibbaren verschieben sollte. Selbst hat er Erfahrungen in der Verschreibung von Buprenorphin, jedoch muss er heute auf dessen Einsatz verzichten, weil Buprenorphin vor ein paar Jahren auf die Liste der verbotenen Substanzen gesetzt wurde. Er selbst bezeichnet seine Methode als «treatment out of desperation». Als nach dem Afghanistankrieg die Drogenabhängigen in Russland schlagartig zugenommen hatten, habe er nach einer Methode gesucht, trotz des Verbots der gängigen Substitutionsmedikamente für einen Teil der Drogenabhängigen eine Behandlung anzubieten.

Als Arzt, der noch zur Sowjetzeit studiert hatte, war er automatisch Offizier der Roten Armee. Er blieb bei der Armee, und im Rang eines Oberstleutnants war er als Psychiater an Forschungen des Militärs zur Beeinflussung des menschlichen Geistes beteiligt. Die sogenannte Zobin-Methode ist ein Nebenprodukt dieser Forschung. Noch heute ist sein Ambulatorium, wo er die Behandlungen durchführt, in einem Gebäude eines Militärspitals untergebracht, auch wenn es als privatwirtschaftliches Unternehmen geführt wird.

Zobin ist nur das öffentliche Aushängeschild der Methode, dahinter steckt noch etwa ein Dutzend weitere Wissenschaftler. Das Verfahren hat das nicht einstimmige Plazet eines akademischen Review board. Zobin ist sich sehr wohl bewusst, dass er seine Daten gemäss einem westlichen Paradigma publizieren müsste. Er hofft, in den nächsten Jahren die Erlaubnis der Forschungsgruppe für eine Publikation zu erhalten.

\section{Mehrstufiges Verfahren}

Über das Verfahren selbst kann ich nur berichten, was ich als Beobachter wahrgenommen habe. Am ersten Tag der Prozedur wurde der Patient mehrere Stunden über Opiatsucht und darüber, wie die Behandlung dagegen wirkt, informiert. Ziel dieser Behandlung ist es, ein Neuropeptid an die Opiatrezeptoren zu binden und dadurch die kranken Rezeptoren auszuschalten. Das Neuropeptid dürfte dabei als nicht kompetitiver Antagonist wirken. Der Patient wurde explizit darauf hingewiesen, was die weiteren Bedingungen für den Erfolg der Kur sind, damit die Sucht wirklich überwunden werden kann. Das
Kernstück dabei sind die drei Ratschläge: 1 . Beziehungen überdenken und Kontakte zur Drogenszene aufgeben; 2. am Morgen nicht im Bett liegenbleiben und einer geregelten Aktivität nachgehen; 3. Sport treiben, um die Endorphine zu stimulieren. Dazu gehören noch die Warnung, jeglichen Heroinkonsum bleibenzulassen, weil ein Rückfall nach der Behandlung eine erhöhte Gefahr für tödliche Überdosierungen berge, und der Hinweis, welche Opioide nicht konsumiert werden dürfen, weil sie die Neuropeptidblockierung der Rezeptoren erodieren. Da es sich hierbei um Opiatagonisten mit schwacher Aktivität handelte, war dies ein Hinweis, wie man gleichwohl einen Rückfall machen könnte.

Opiatabhängigkeit ist nach Zobins Modell primär (aber nicht ausschliesslich) ein Geschehen an den Opiatrezeptoren. Kurz zusammengefasst verändern sich in der Abhängigkeit die Rezeptoren in Struktur und Anzahl, und durch die wiederholte externe Stimulation durch Opiate treten «Kindling»-Effekte auf (ein Begriff aus der Epileptologie, der in einer westlichen Terminologie in Zusammenhang mit Sucht als LTP [long-term potentiation] bezeichnet wird). Die veränderten Rezeptoren bildeten dann eine «Matrix», die es den vermindert produzierten Endorphinen verunmöglicht, normal zu wirken. Die therapeutische Strategie sieht vor, mit einer injizierten klaren Flüssigkeit sowie Elektro- und Magnetstimulation die «Matrix» zu durchbrechen, um die Bindung des Neuropeptids, das die Rezeptoren abdecken soll, zu ermöglichen.

Der Patient erhielt nach der mehrstündigen Information nochmals eine Nacht Bedenkzeit. Das Verfahren wurde am zweiten Tag durch intravenöse Injektion der klaren Flüssigkeit eingeleitet. Gleichzeitig erfolgte eine frontookzipitale transkraniale Elektrostimulation, und Zobin applizierte Magnetimpulse über den frontalen und parietalen Regionen des Schädels. Der Patient stand dabei unter der Überwachung eines Anästhesisten. Er war an einen modernen Siemens-Monitor zur Überwachung der Vitalparameter und an einen zweiten Computermonitor zur Überwachung des Verfahrens selbst angeschlossen. Nachdem am Computermonitor die kyrillisch beschrifteten Parameter bestimmte Werte erreichten, wurde eine milchige Flüssigkeit injiziert, das Neuropeptid.

Klinisch zeigte der Patient nach Einleiten der Behandlung einen Flush am ganzen Körper, einen Pulsanstieg, und er geriet in einen tranceartigen Zustand, der etwa eine halbe Stunde dauerte. Die anschliessenden Gespräche mit Zobin ergaben, dass es sich bei der klaren Flüs- 
** Zobin belegt damit, dass auch die Sowjets wie die USA - wie man spätestens seit dem Bekanntwerden des Falls Olson weiss mit Psychedelika im militärischen Einsatz forschten. Der CIA-Agent Olson «fiel» nach einem LSD Experiment der CIA aus einem Hotelfenster. Die Nachforschungen seines Sohnes ergaben, dass Olson wohl aus dem Fenster des Hotels geworfen wurde, weil er drohte, bakterielle Freisetzungsversuche in Korea der Öffentlichkeit mitzuteilen. Leider sind die Resultate dieser Forschungen beider Länder der Öffentlichkeit nicht zugänglich.

\section{Literatur}

1 Stohler R, Moldovanyi A. Die neue Wunderkur für Heroinabhängige - Dr. Zobins «Transmodulationsblockade». Schweiz Ärztezeitung 2006;87(14):610-1.

2 Пятницкая ИН, Зобин М . Короткая амбулаторная программа лечения опийной наркомании в раннем постабстинентном периоде. Наркология 2004;(6):52-6. (Pjatnizkaja IN, Zobin ML. Ambulante Kurzbehandlung von Opiatabhängigen in der frühen Postabstinenzperiode. Narkologija 2004;[6]:52-6) Ein (russisches) Abstract des Artikels findet sich auf: www.narkotiki.ru/narkologia ar2004.html. sigkeit, wie ich vermutet hatte, um ein Psychedelikum handelte. Den Namen dürfe er mir zum jetzigen Zeitpunkt nicht sagen, da dieser immer noch unter militärischer Geheimhaltung stehe. Aufgrund der Wirkdauer dürfte es sich aber um ein kurzwirksames Tryptamin gehandelt haben.**

Am dritten Tag erfolgte der Expositionsversuch mit Meperidin, nachdem die peripheren Opiatrezeptoren mit Loperamid abgedeckt worden waren. Der dadurch ausgelöste Atemstillstand verlief auf eine für Opioide unübliche Weise, indem der Patient eine generelle Muskellähmung bei erhaltenem Bewusstsein und normaler Pupillenreaktion erlebte. Ob es sich wirklich um eine Exposition mit Meperidin handelte, konnte ich nicht überprüfen. Der Patient wurde während des Atemstillstandes kurze Zeit mechanisch beatmet, die Sauerstoffsättigung fiel nie unter $95 \%$, und der Anästhesist war für eine allfällige Intubation vorbereitet.

\section{Keine Scharlatanerie}

Wie ist die Sache nun zu beurteilen? Mit Sicherheit lässt sich sagen, dass Zobin kein erratischer Wirrkopf ist, der Wunderheilungen verspricht. Er ist in eine Schulmedizin eingebunden, zu der unsere Schulmedizin schlechten Zugang findet, die sich aber wie unsere auf Forschungen abstützt. Zobin selbst sieht seine Behandlungsmethode als adjuvante Therapie zu einer Langzeitentwöhnung, die auch nach unserem Verständnis zum Ausstieg aus einer Opiatabhängigkeit nötig ist. Die Anforderungen, um in die Behandlung aufgenommen zu werden, sind hoch: reine Opiatabhängigkeit, absolvierter Opiatentzug und anschliessend mindestens dreiwöchige Abstinenz, Begleitperson während der Behandlung und hohe Motivation. Zudem dürften die erwähnten Kosten prohibitiv wirken, wobei der Betrag von etwa Fr. 15000 den Gesamtkosten und nicht dem Betrag für die Behandlung entspricht (Behandlung plus zwei Flüge und Hotelunterkunft für zwei Personen). Entsprechend kommt nur ein verschwindend kleiner Teil der schweizerischen Drogenabhängigen für diese Behandlung überhaupt in Frage. Angehörige und Behörden können also nicht einfach die Abhängigen nach Moskau schicken, um sie dort heilen zu lassen. Dass die Behandlung zur Wunderkur stilisiert wird, ist ein in der Schweiz gemachtes Problem. Die gleichen Kreise, die mit ihrer Initiative «Jugend ohne Drogen» baden gingen, versuchen nun, Zobins Methode als Alternative zu den Substitutionstherapien zu propagieren. Sie hätten wenig Freude daran, zu hören, wie Zobin sich derzeit engagiert, um Methadon für die
Behandlung der Abhängigen in Russland einzuführen.

Schädlichkeit und Wirkung sind natürlich nach einem Besuch nicht abschliessend zu beurteilen. Zobin hat bis heute schon über 2000 russische Abhängige mit der Methode behandelt, und wenn daraus unerwartete Schädigungen resultieren würden, ist anzunehmen, dass ihn die russischen Behörden schon längst gestoppt hätten.

Am Morgen nach der Behandlung berichtete der Patient, den wir begleiteten, dass er seit 20 Jahren nicht mehr so gut geschlafen habe. Gemäss den Berichten von Menschen, die die Behandlung gemacht haben, besteht deren Wirkung hauptsächlich darin, dass die nach einem Entzug latent ständig vorhandenen Gedanken an einen erneuten Heroinkonsum verschwanden. Liesse sich dies verifizieren, wäre es sehr interessant. Unser Behandlungssystem hat bei diesen für die Patienten quälenden Zuständen leider nichts anzubieten.

Bei der Behandlung war offensichtlich, dass Zobin stark mit Bedeutungen arbeitete, die psychotherapeutischen Aspekte seiner Arbeit habe ich aber mit Zobin kaum diskutiert. Die Strategie ist jedoch in einem Artikel von Pjatnizkaja und Zobin in der Fachzeitschrift «Narkologija» nachzulesen [2]. Sie bezeichnen das Verfahren als eklektische Kurztherapie, die Elemente der emotional-rationalen Verhaltenstherapie nach A. Ellis, Konfrontationsstrategien nach G. Garner und eines Konzepts von A. N. Leontjew beinhaltet. Dabei geht es darum, motivierten Patienten eine psychobiologische Deutung ihrer Abhängigkeit zu geben, um die Diskrepanz zwischen ihrer Krankheit und ihren Ressourcen zu verstärken. Durch Konfrontation sollen Verhaltensstereotypien als negativ identifiziert, durch Manipulationstechniken eine Stimmung der radikalen Hilfe geschaffen und eine Strategie der positiven Überwindung ohne Alternativen aufgezeigt werden. Ziel ist es zudem, die Verantwortung für den Erfolg vom Arzt auf die Patienten zu übertragen. Die Umorientierung der Patienten wird durch das dramatische Tiefenerlebnis der biologischen Behandlung unterstützt. Die Kontrolle am dritten Tag hat in diesem Kontext eine aversive Bedeutung. Die Autoren sind der Meinung, dass die Patienten danach während zweier, dreier Jahre weitere Unterstützungen in verschiedenen Bereichen im Sinne von Motivationsstützen benötigen. Sie favorisieren dabei einen systemischen Ansatz.

Im Artikel werden auch einige Verlaufsdaten zu 435 Behandlungen dargestellt. 